\title{
Medication knowledge of patients hospitalized for heart failure at admission and after discharge
}

This article was published in the following Dove Press journal:

Patient Preference and Adherence

9 November 2016

Number of times this article has been viewed

\section{Florian Custodis' \\ Franziska Rohlehr' \\ Angelika Wachter' \\ Michael Böhm' \\ Martin Schulz ${ }^{2}$ \\ Ulrich Laufs'}

'Department of Internal Medicine III, Cardiology, Angiology and Intensive Care Medicine, Saarland University Medical Center, Homburg/Saar, ${ }^{2}$ Department of Clinical Pharmacy and Biochemistry, Institute of Pharmacy, Freie Universitaet Berlin, Berlin, Germany
Correspondence: Ulrich Laufs Department of Internal Medicine III, Cardiology, Angiology and Intensive Care Medicine, Saarland University Medical Center (IMED), Kirrberger Straße, 6642I Homburg/Saar, Germany $\mathrm{Tel}+49684 \mid \quad 16 \quad 15350$ Fax +49 684II6 I5I75 Email ulrich@laufs.com
Background: A substantial aspect of health literacy is the knowledge of prescribed medication. In chronic heart failure, incomplete intake of prescribed drugs (medication non-adherence) is inversely associated with clinical prognosis. Therefore, we assessed medication knowledge in a cohort of patients with decompensated heart failure at hospital admission and after discharge in a prospective, cross-sectional study.

Methods: One hundred and eleven patients presenting at the emergency department with acute decompensated heart failure were included (mean age 78.4 $99.2,59 \%$ men) in the study. Patients' medication knowledge was assessed during individual interviews at baseline, course of hospitalization, and 3 months after discharge. Individual responses were compared with the medical records of the referring general practitioner.

Results: Median N-terminal prohormone of brain natriuretic peptide plasma concentration in the overall population at baseline was $4,208 \mathrm{pg} / \mathrm{mL}(2,023-7,101 \mathrm{pg} / \mathrm{mL}$ [interquartile range]), 20 patients died between the second and third interview. The number of prescribed drugs increased from $8 \pm 3$ at baseline to $9 \pm 3$ after 3 months. The majority of patients did not know the correct number of their drugs. Medication knowledge decreased continuously from baseline to the third interview. At baseline, $37 \%(\mathrm{n}=41)$ of patients stated the correct number of drugs to be taken, whereas only $18 \%(\mathrm{n}=16)$ knew the correct number 3 months after discharge $(P=0.008)$. Knowledge was inversely related to $\mathrm{N}$-terminal prohormone of brain natriuretic peptide levels.

Conclusion: Medication knowledge of patients with acute decompensated heart failure is poor. Despite care in a university hospital, patients' individual medication knowledge decreased after discharge. The study reveals an urgent need for better strategies to improve and promote the knowledge of prescribed medication in these very high-risk patients.

Keywords: medication knowledge, hospitalization, chronic heart failure, health literacy

\section{Introduction}

Chronic heart failure $(\mathrm{CHF})$ is a serious health care problem affecting $1 \%-2 \%$ of the adult population in developed countries with the prevalence rising to $\geq 10 \%$ among persons $\geq 70$ years of age. ${ }^{1}$ Evidence-based pharmacotherapy has improved prognosis in patients with systolic heart failure. ${ }^{2}$ Adherence to these medications, defined as the extent to which patients take medications as prescribed, prevents hospitalization and mortality.,

Management of chronic diseases, including heart failure, requires a high level of self-care skills that are determined by patients' health literacy, which is defined as the degree to which patients have the capacity to adapt health information and services needed to make appropriate health decisions. ${ }^{4}$ A substantial aspect of health literacy represents the knowledge of one's medical condition and treatment regimens, especially daily medication. ${ }^{5}$ Medication knowledge was shown to determine medication adherence in CHF patients and is inversely associated with outcomes in CHF. ${ }^{6,7}$ Lack 
of patients' knowledge about their discharge medication increases the risk of drug-related problems. ${ }^{4}$ During hospitalization, established medication regimens are frequently modified, and in $>75 \%$ of cases, even three or more alterations are carried out. ${ }^{8} \mathrm{~A}$ fact that represents a challenge for the interaction between health care providers and patients.

Cognitive functioning is impaired in patients with decompensated heart failure. It improves but does not normalize after recompensation ${ }^{9,10}$ Several studies characterized health literacy and medication knowledge in CHF populations either at the time of hospitalization or after discharge. However, a potential alteration of knowledge over time from acute hospital admission to the outpatient setting and a potential role of recompensation as an effector of knowledge have not been investigated. We hypothesized an increase in the individual medication-related knowledge during the course of treatment. Therefore, the objective of the present study was to quantify medication knowledge in a cohort of patients referred to and discharged from a tertiary clinical center due to decompensated heart failure.

\section{Methods}

\section{Patients}

This evaluation is a prospective, cross-sectional study. We consecutively enrolled 111 patients between 54 and 98 years of age who had been hospitalized for decompensated heart failure at the emergency department of the Saarland University Hospital. The following inclusion criteria were required: acute decompensated heart failure, established medical therapy, and an ability to give written consent and participate in the interview. CHF was defined according to the European Society of Cardiology guidelines. ${ }^{2}$ After written informed consent was obtained, the patients were interviewed by an independent data collector not involved in patient care. The study was approved by the local ethics committee ("EthikKommission der Ärztekammer des Saarlandes") (identification number 98/13) in accordance with the Declaration of Helsinki. All patients gave their written informed consent to participate in the study.

\section{Clinical and demographic variables}

Demographic characteristics (sex, age), body mass index, marital status, degree of education, New York Heart Association functional class for dyspnea, cardiovascular (CV) comorbidities, and admitting diagnosis were assessed (Table 1). Heart rate and blood pressure were measured at baseline after resting for at least 5 minutes. N-terminal prohormone of brain natriuretic peptide (NT-proBNP) levels were measured at baseline. The number and type (CV and
Table I Baseline characteristics of the study population ( $\mathrm{N}=$ I I I)

\begin{tabular}{|c|c|}
\hline \multicolumn{2}{|l|}{ Demographics } \\
\hline Age (years $\pm S D)$ & $78.4 \pm 9.2$ \\
\hline Female (n) & $41 \%(46)$ \\
\hline \multicolumn{2}{|l|}{ Cardiac parameters (mean \pm SD) } \\
\hline $\mathrm{SBP}(\mathrm{mmHg})$ & $137 \pm 25$ \\
\hline $\mathrm{DBP}(\mathrm{mmHg})$ & $76 \pm 16$ \\
\hline Heart rate (bpm) & $83 \pm 22$ \\
\hline NYHA (latest known before hospitalization) & $3.2 \pm 0.8$ \\
\hline NTproBNP (pg/mL) (median + interquartile range) & $4,208(2,023-7,101)$ \\
\hline $\mathrm{BMI}\left(\mathrm{kg} / \mathrm{m}^{2}\right)$ & $29.1 \pm 5.8$ \\
\hline \multicolumn{2}{|l|}{ Medical history (n) } \\
\hline Hypertension & $52 \%(58)$ \\
\hline Coronary heart disease & $50 \%(56)$ \\
\hline Atrial fibrillation & $47 \%(52)$ \\
\hline Chronic kidney disease & $36 \%(40)$ \\
\hline Diabetes type II & $29 \%(32)$ \\
\hline COPD & $10 \%(I I)$ \\
\hline Number of daily medications (mean \pm SD) & $8 \pm 3$ \\
\hline \multicolumn{2}{|l|}{ Education (n) } \\
\hline $0-9$ years & $78 \%(86)$ \\
\hline 10 years & $7 \%(8)$ \\
\hline 13 years & $15 \%(17)$ \\
\hline \multicolumn{2}{|l|}{ Occupation (n) } \\
\hline Homemaker & $16 \%(18)$ \\
\hline Non-qualified manual labor & $19 \%(2 I)$ \\
\hline Qualified manual labor & $56 \%(62)$ \\
\hline University graduate & $9 \%(10)$ \\
\hline \multicolumn{2}{|l|}{ Relationship status (n) } \\
\hline Living alone & $44 \%(49)$ \\
\hline In relationship & $56 \%(62)$ \\
\hline
\end{tabular}

Abbreviations: BMI, body mass index; BNP, brain natriuretic peptide; COPD, chronic obstructive pulmonary disease; DM II, diabetes mellitus type 2; NYHA, New York Heart Association; SD, standard deviation; SBP, systolic blood pressure; DBP, diastolic blood pressure.

non- $\mathrm{CV}$ ) of medicines prescribed were documented and verified from the patient chart at admission (baseline = interview 1) and during hospitalization at days $2-4$ (second interview). At home, 3 months (mean 106 days) after discharge, the prescribed medication was verified by comparison with the outpatient record of the treating general practitioner.

\section{Patient-reported medication knowledge}

Fifteen types of prescribed CHF-related drugs were categorized (Table 2). To determine patients' knowledge about their medication, the following questions were asked: How many different medications do you take? How many heartspecific medications do you take? Do you know the name (generic or brand)? Do you know the function/mode of action? Do you know the dosage form? Do you know when to take the medications? The patients were asked these questions at the time of hospitalization in the emergency department (baseline), in the hospital ward after stabilization on days 2-4 (second interview) and at home, 3 months after 
Table 2 Knowledge of prescribed HF-related drug classes (n: patient knew drug type)

\begin{tabular}{llll}
\hline Type of drug & \multicolumn{3}{l}{ Knowledge (n/total pt with drug) } \\
\cline { 2 - 4 } & Baseline \% & Hospital \% & $\mathbf{3}$ months \% \\
\hline Loop diuretics & $38(34 / 89)$ & $38(33 / 86)$ & $49(40 / 82)$ \\
B-Blockers & $28(24 / 87)$ & $29(24 / 83)$ & $29(22 / 75)$ \\
ACE inhibitors/ATI-receptor & $37(3 / / 84)$ & $35(28 / 80)$ & $28(21 / 76)$ \\
antagonists & & & \\
ASA & $5 \mathrm{I}(24 / 47)$ & $56(25 / 45)$ & $67(22 / 33)$ \\
OAC (phenprocoumon) & $83(29 / 35)$ & $91(30 / 33)$ & $70(26 / 37)$ \\
Thiazides & $42(13 / 31)$ & $46(13 / 28)$ & $29(7 / 24)$ \\
Calcium antagonists & $23(7 / 30)$ & $24(7 / 29)$ & $32(7 / 22)$ \\
Potassium-sparing diuretic & $29(6 / 21)$ & $32(6 / 19)$ & $34(12 / 35)$ \\
Antiplatelet drugs & $8(1 / 12)$ & $8(1 / 12)$ & $20(1 / 5)$ \\
Vasodilatators & $17(2 / / 2)$ & $17(2 / 12)$ & $21(3 / 14)$ \\
Antiarrhythmics & $45(5 / 11)$ & $50(5 / 10)$ & $45(5 / / 1)$ \\
Digitalis & $13(1 / 8)$ & $13(1 / 8)$ & $0(0 / 6)$ \\
NOACs & $38(3 / 8)$ & $50(4 / 8)$ & $67(8 / 12)$ \\
Antihypertensive drugs & $67(4 / 6)$ & $50(3 / 6)$ & $33(1 / 3)$ \\
(other) & & & \\
Antianginal drugs & $0(0 / 3)$ & $0(0 / 3)$ & $25(1 / 4)$ \\
\hline
\end{tabular}

Abbreviations: $A C E$, angiotensin-converting enzyme; ASA, acetylsalicylic acid; $\mathrm{HF}$, heart failure; NOACs, novel oral anticoagulants; pt, patients.

discharge (third interview). Knowledge at baseline and at the second interview refers to the medication taken before hospitalization. Knowledge at the third interview refers to medication taken according to the general practitioner's record in the outpatient setting.

\section{Statistics}

This evaluation is a descriptive, cross-sectional study. Data are presented as mean \pm standard deviation (SD) of the mean and their distribution as percentages unless otherwise specified. Baseline characteristics were presented using descriptive statistics with mean (SD) for quantitative variables, and numbers and percentages for categorical variables. Significance tests were repeated-measures analysis of variance using within-subjects factor group (hospital admission, hospital stay, and 3-month follow-up) including post hoc Bonferroni tests to assess individual differences with respect to medication knowledge. Values of $P<0.05$ were considered significant. All statistical analyses were calculated using IBM SPSS statistics software (version 23.0; IBM Corporation, Armonk, NY, USA).

\section{Results}

\section{Patients}

The baseline characteristics of the 111 patients included in the study are summarized in Table 1 . The mean age was $78.4 \pm 9.2$ years, $59 \%$ were males, body mass index $29.1 \pm 5.8 \mathrm{~kg} / \mathrm{m}^{2}, 56 \%$ were married/in relationship. On average, patients were categorized as New York Heart Association class III prior to hospitalization. Mean heart rate at hospital admission was $83 \pm 22 \mathrm{bpm}$. Mean systolic blood pressure was $137 \pm 25 \mathrm{mmHg}$. Median NT-proBNP plasma concentration in the overall population at baseline was $4,208 \mathrm{pg} / \mathrm{mL}$ (2,023-7,101 pg/mL [interquartile range]). The most common comorbidities were arterial hypertension (52\%), coronary heart disease (50\%), atrial fibrillation (47\%), chronic kidney disease (36\%), diabetes mellitus (29\%), and chronic obstructive pulmonary disease (10\%). Of the 111 patients included in the study, 20 died between the second and third interview.

\section{Number and types of prescribed drugs}

At baseline, the patients took, on average, $4 \pm 2$ prescribed drugs out of the $15 \mathrm{CV}$ drug classes (Table 2). The average number of totally prescribed drugs was $8 \pm 3$ per person. After 3 months, each patient took $5 \pm 1$ prescribed drugs out of the $15 \mathrm{CV}$ drug classes (totally number of daily medications was $9 \pm 3$ per person). Hence, the number of drugs to be taken increased by an average of one per person due to additional prescription during hospitalization. Of the different drug classes, oral anticoagulation (OAC)/VKA (phenprocoumon) and aspirin were the best known (Table 2).

\section{Knowledge of the number of daily medications}

To assess the knowledge of the number of drugs taken, the number stated by the patient was compared to the prescribed number and calculated as percent deviation. Knowledge of the correct number of daily medications assessed at baseline and at home, 3 months after discharge is shown in Figure $1 \mathrm{~A}$ and $\mathrm{B}$.

In contrast to our expectation, medication knowledge decreased continuously from baseline to the third interview. At baseline, 41 patients (37\%) stated the correct number of drugs taken, whereas only 16 patients (18\%) knew the correct number after discharge $(P=0.008)$. Figure 2 shows that the number of patients underestimating the number of drugs particularly increased after discharge. The number of patients exhibiting a deviation of $100 \%$ increased from six patients at baseline to 14 patients at the third interview. Figure 3 illustrates the amount of underestimation of the correct number of medications.

\section{Knowledge of the number of daily medications based on NT-proBNP levels and age}

To assess individual knowledge of heart-specific medication (correct number, name [generic or brand] out of the $15 \mathrm{CV}$ drug classes, mode of action, dosage form, time-points of 

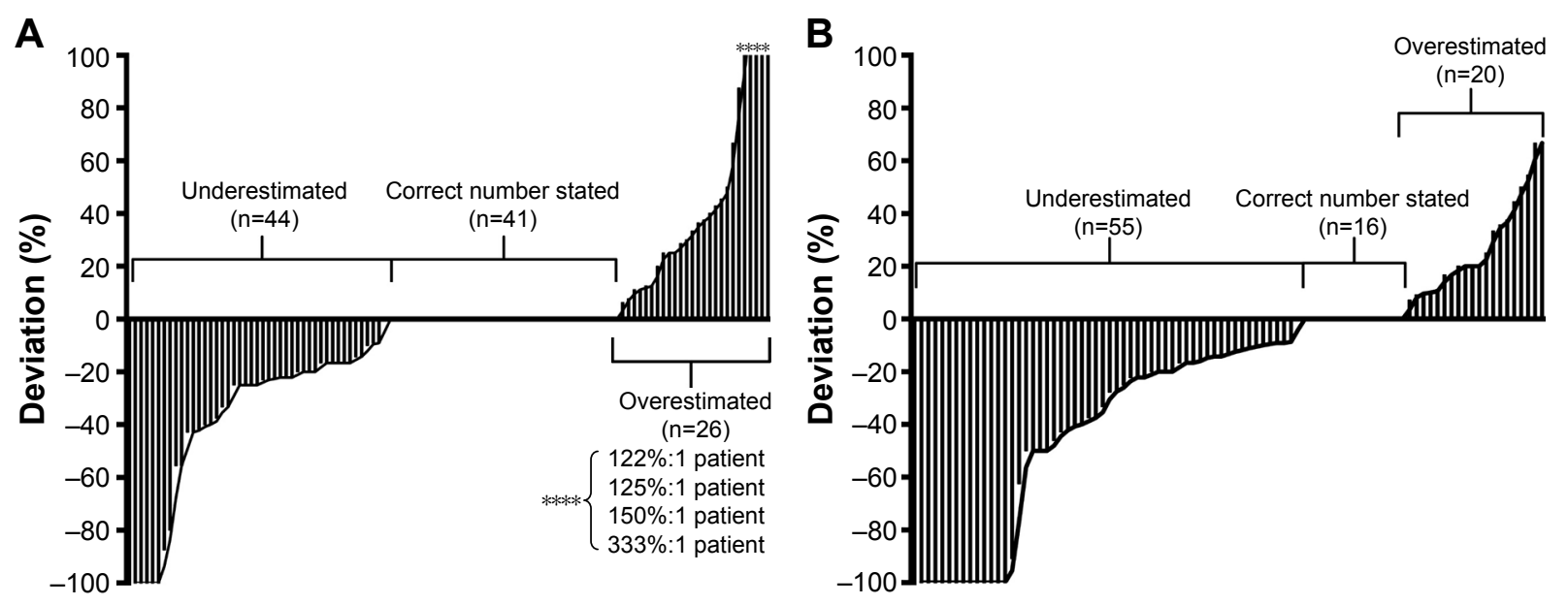

Figure I (A) Patients' knowledge of the number of drugs compared to approved number of prescribed drugs at home (baseline). Each line indicates one patient $(\mathrm{n}=\mathrm{II}$ I). (B) Patients' knowledge of the number of drugs compared to approved number of prescribed drugs at home (3 months). Each line indicates one patient ( $\mathrm{n}=9 \mathrm{I})$. $0 \%$ deviation $=$ correct number of drugs stated.

Note: *****Four patients had a percentage of more than 100

administration) in relation to the severity of heart failure and age, patients were stratified according to NT-proBNP and age tertiles. An inverse relation between medication knowledge and NT-proBNP levels was found at all three interviews (timepoints) as percent knowledge of the correct number of drugs decreased from tertile 1 to tertile 3 (Figure 4). Patients within the second age tertile (76-82 years) exhibited the best knowledge compared to older or younger individuals (Figure 5).

\section{Discussion}

The main finding of this study was that the individual level of knowledge regarding heart failure-related medication was low in a cohort of CHF patients hospitalized for decompensated heart failure and was inversely related to the severity of the disease but not with regard to age. In contrast to our expectations, the knowledge decreased even further from the time of hospital admission to the outpatient setting.

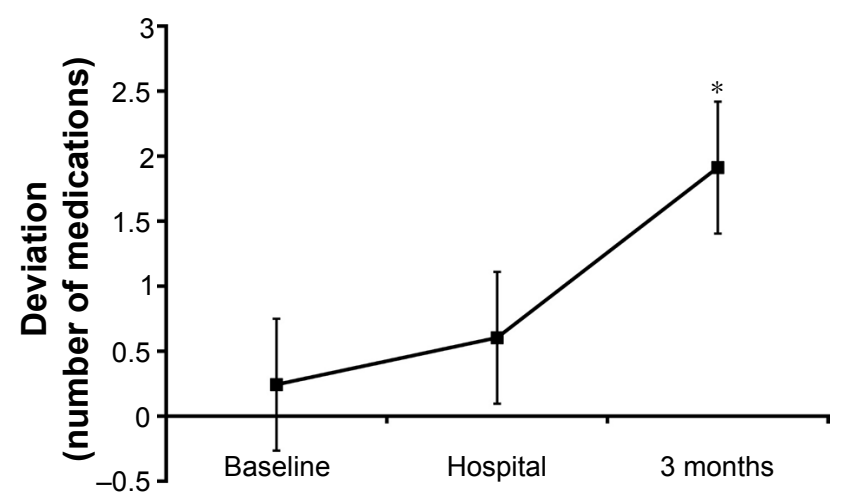

Figure 2 Deviation of stated number of daily medications compared to prescribed number of daily medications.

Notes: Mean \pm SE. ${ }^{*} P<0.05$ knowledge at 3 months vs baseline (interview I). Abbreviation: SE, standard error.
CHF is one of the most important causes of morbidity and mortality in Western countries, and symptoms related to $\mathrm{CHF}$ are the leading cause of hospitalization, ${ }^{11,12}$ contributing to $70 \%$ of the total treatment-related costs in patients aged $>65$ years, representing $\sim 2 \%$ of the total health-care expenditure in industrialized countries. ${ }^{13}$ Patients with $\mathrm{CHF}$ require polypharmacy to control symptoms, slow disease progression, and decrease hospitalization and mortality. ${ }^{2}$ Health literacy, self-care skills, and, in particular, knowledge of one's medication are a prerequisite for sustained medication adherence. Low literacy is associated with adverse outcomes in heart failure, including increased risk of hospitalization. ${ }^{4}$

Hospitalization for heart failure is closely and inversely linked to outcome in patients with chronic heart failure. ${ }^{14}$ An important predictor of rehospitalization among other patient-related factors is medication non-adherence, which may account for up to one-third of hospitalizations. ${ }^{15}$

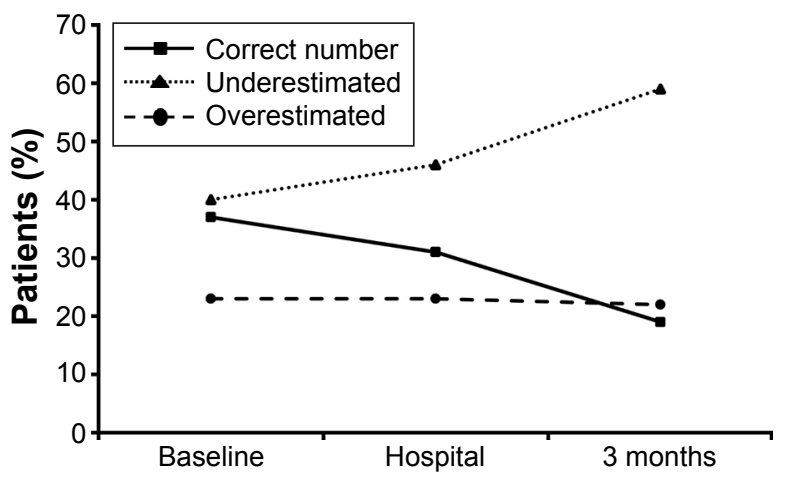

Figure 3 Patients' knowledge of the number of drugs. Percent of patients stating the correct number of drugs, over- and underestimating the number of drugs compared to prescribed (\%). 


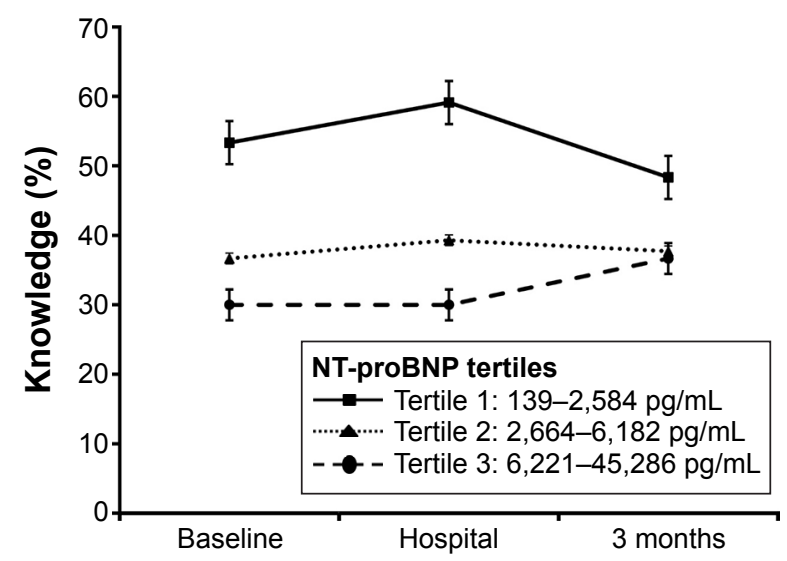

Figure 4 Medication knowledge depending on NT-proBNP level tertiles at the time of hospitalization (baseline). Percent of correct answers (correct number, name [generic or brand], mode of action, dosage form, time-points of administration) at baseline $(n=|| I)$, at days $2-4$ of hospital stay $(n=106)$, and 3 months after discharge from the hospital $(n=91)$ of surviving patients based on NTproBNP values at baseline. Median $\pm \mathrm{SE}$.

Abbreviations: SE, standard error; NT-proBNP, N-terminal prohormone of brain natriuretic peptide.

In our cohort, medication knowledge was lower than expected. Data from previous studies are heterogeneous and not easily comparable due to differences of the design and methodology. In a Swedish study population of elderly CHF patients, $55 \%$ could correctly name what medication had been prescribed 1 month after prescription. ${ }^{16}$ In a population of patients discharged from an internal medicine residency service at a community-based teaching hospital, $86 \%$ were aware that they had been prescribed new medicines, but fewer could correctly identify the name $(64 \%)$ or number $(74 \%)$ of new drugs in an interview between 4 and 18 days after discharge. ${ }^{17}$ Interestingly in our study, a lack of knowledge

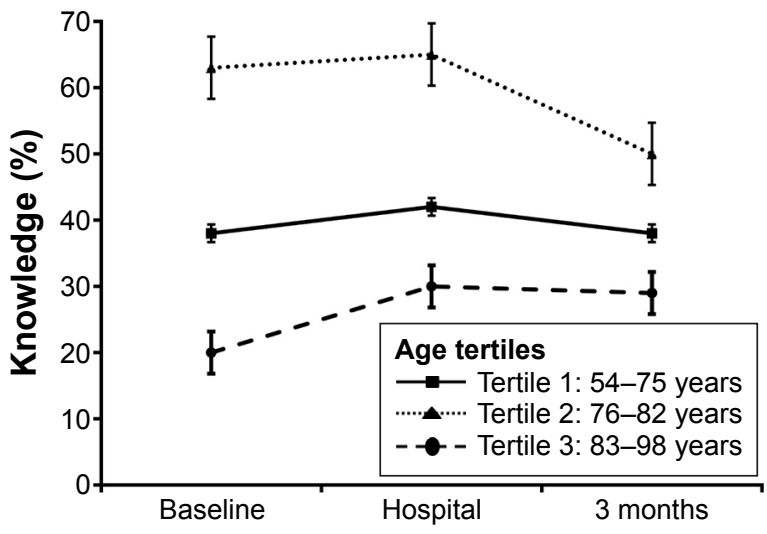

Figure 5 Medication knowledge depending on age tertiles. Percent of correct answers (correct number, name [generic or brand], mode of action, dosage form, time-points of administration) at baseline ( $\mathrm{n}=\mathrm{III})$, at days 2-4 of hospital stay $(n=106)$, and 3 months after discharge from the hospital $(n=91)$ of surviving patients based on age tertiles. Median $\pm \mathrm{SE}$.

Abbreviation: SE, standard error. was not related to older age, a finding that is indirectly supported by a recent literature review, suggesting that older age alone is not related to poorer medication adherence in patients with CHF. ${ }^{18}$

Reasons for low health literacy and lack of knowledge are multifactorial and may even be a proxy for other determinants such as cognitive impairment, severity of disease, socioeconomic characteristics, and other barriers to sufficient health care that are associated with poor outcomes. ${ }^{4}$ Apart from such patient-related factors, other factors related to health care providers (eg, communication strategies) are crucial and determine patients' knowledge.

Patients in our study received standardized advice regarding the type, dosage, and purpose of discharge medication by the attending physician at the time of discharge. During the outpatient course, follow-up visits were provided by office-based cardiologists and general practitioners pursuing individual counseling strategies. Thus, the knowledge deficits observed may result from a lack of an educational strategy implemented in the clinical routine. Though the role for patient counseling and education seems obvious, health care providers tend to lose focus and - maybe even more important - are often unaware of literacy problems or overestimate patients' understanding of health information and knowledge. Thus, appropriate strategies beyond standard instructions are not implemented. ${ }^{4}$

A variety of strategies and interventions focusing on education to promote patients' health literacy and knowledge can be found in the literature and are highlighted by the current heart failure guidelines and consensus documents. ${ }^{2,4}$ Education was shown to improve knowledge, self-monitoring, medication adherence, time to hospitalization, and reduced days spent in the hospital. ${ }^{19}$ Patients who receive structured in-hospital education have higher knowledge scores at discharge and 1 year later when compared with those who did not receive in-hospital education. ${ }^{20}$ Among the strategies to improve recall of medical advice, complementing verbal information with written instructions improved medication recall in a broad spectrum of patient populations. ${ }^{21-23}$ Implementation of an enhanced medication plan as an adjunct to the discharge conversation between physician and patient improved information transfer and patients' knowledge of their drug treatment in a cohort of patients from several internal clinics in a university hospital. Interestingly, the intervention did not prolong the overall discharge process. ${ }^{21}$ Although, to date, a clear survival benefit of in-hospital or discharge education could not be demonstrated, prior data have suggested that discharge education may result in fewer 
days of hospitalization, lower costs, and lower mortality rates within a 6-month follow-up. ${ }^{24}$

Previous studies in CHF patients like CogImpair-HF documented changes in cognitive function, which improved after recompensation. ${ }^{9}$ In contrast, in our study, individual medication knowledge decreased over time. This may be due to differences in the study design. In the CogImpair-HF study, patients where reevaluated after successful recompensation $14 \pm 7$ days after the index evaluation. In our study, patients were questioned on days 2-4 and after 3 months. At least, the time of the latter interview might have been postponed too far from the index event, leaving room for potential renewed decompensation and associated gaps of knowledge. On the other hand, the repeated interview itself may confer a training effect; however, this was clearly not sufficient to improve medication knowledge.

There are some limitations of this investigation. One is the relatively small sample size, which may not be regarded as representative of the overall heart failure population. Future studies with appropriate sample sizes are needed to confirm our study results. Another point is that we did not assess potential and clinically relevant cognitive disorders (eg, depression and dementia) before inclusion. Such disorders are highly prevalent in heart failure and play a central role for patient-related adherence. ${ }^{25}$ Therefore, we cannot rule out completely that cognitive impairments associated with cardiac decompensation may have been confounded with symptoms of neurocognitive disorders. Due to structural limitations, it was not feasible to apply dedicated cognitive tests to measure the complete range of cognitive functions in our study. This point should be addressed by dedicated research protocols in future studies.

\section{Conclusion and implications}

In this observational study, we documented a low level of medication-associated knowledge in a contemporary cohort of $\mathrm{CHF}$ patients. In contrast to our hypothesis, knowledge decreased over time despite hospital care. The main reason for these findings may be found in the absence of structured counseling during hospitalization at discharge and during ambulatory care. Therefore, a central necessity is the recognition of the individual extent of patients' health literacy and to identify patients at risk for low literacy, which may affect and compromise even basic skills. Moreover, this study underpins the need for focused patient counseling during hospitalization, at discharge, and within the outpatient setting regarding seamless pharmacotherapy in CHF patients. In order to reliably continue these therapies after discharge, continuous and structured communication between clinicians, primary care physicians, and patients is essential. Additional and innovative strategies addressing medicine management in this high-risk population are urgently needed.

\section{Disclosure}

The authors report no conflicts of interest in this work.

\section{References}

1. Mosterd A, Hoes AW. Clinical epidemiology of heart failure. Heart. 2007;93(9):1137-1146.

2. McMurray JJ, Adamopoulos S, Anker SD, et al; ESC Committee for Practice Guidelines. ESC Guidelines for the diagnosis and treatment of acute and chronic heart failure 2012: The Task Force for the Diagnosis and Treatment of Acute and Chronic Heart Failure 2012 of the European Society of Cardiology. Developed in collaboration with the Heart Failure Association (HFA) of the ESC. Eur Heart J. 2012;33(14):1787-1847.

3. Fitzgerald AA, Powers JD, Ho PM, et al. Impact of medication nonadherence on hospitalizations and mortality in heart failure. J Card Fail. 2011;17(8):664-669.

4. Evangelista LS, Rasmusson KD, Laramee AS, et al. Health literacy and the patient with heart failure -implications for patient care and research: a consensus statement of the Heart Failure Society of America. J Card Fail. 2010;16(1):9-16.

5. Laufs U, Rettig-Ewen V, Böhm M. Strategies to improve drug adherence. Eur Heart J. 2011;32(3):264-268.

6. van der Wal MH, Jaarsma T, Moser DK, Veeger NJ, van Gilst WH, van Veldhuisen DJ. Compliance in heart failure patients: the importance of knowledge and beliefs. Eur Heart J. 2006;27(4):434-440.

7. Hope CJ, Wu J, Tu W, Young J, Murray MD. Association of medication adherence, knowledge, and skills with emergency department visits by adults 50 years or older with congestive heart failure. Am J Health Syst Pharm. 2004;61(19):2043-2049.

8. Himmel W, Kochen MM, Sorns U, Hummers-Pradier E. Drug changes at the interface between primary and secondary care. Int J Clin Pharmacol Ther. 2004;42(2):103-109.

9. Kindermann I, Fischer D, Karbach J, et al. Cognitive function in patients with decompensated heart failure: the cognitive Impairment in Heart Failure (Cogimpair-HF) study. Eur J Heart Fail. 2012;14(4): 404-413.

10. Fikenzer K, Knoll A, Lenski D, Schulz M, Böhm M, Laufs U. Chronische Herzinsuffizienz: Teufelskreis aus geringer Einnahmetreue von Medikamenten und kardialer Dekompensation [Poor medication adherence and worsening of heart failure - a vicious circle]. Dtsch Med Wochenschr. 2014;139(47):2390-2394. German.

11. Zannad F, Agrinier N, Alla F. Heart failure burden and therapy. Europace. 2009;11(Suppl 5):v1-v9.

12. Alla F, Zannad F, Filippatos G. Epidemiology of acute heart failure syndromes. Heart Fail Rev. 2007;12(2):91-95.

13. Stewart S, MacIntyre K, Capewell S, McMurray JJ. Heart failure and the aging population: an increasing burden in the 21 st century? Heart. 2003;89(1):49-53.

14. Gheorghiade M, Vaduganathan M, Fonarow GC, Bonow RO. Rehospitalization for heart failure: problems and perspectives. $J$ Am Coll Cardiol. 2013;61(4):391-403.

15. Van der Wal MHL, Jaarsma T, van Veldhuisen DJ. Non-compliance in patients with heart failure: how can we manage it? Eur J Heart Fail. 2005;7(1):5-17.

16. Cline CM, Bjorck-Linne AK, Israelsson BY, Willenheimer RB, Erhardt LR. Non-compliance and knowledge of prescribed medication in elderly patients with heart failure. Eur J Heart Fail. 1999;1(2):145-149.

17. Maniaci ML, Heckman MG, Dawson NL. Functional health literacy and understanding of medications at discharge. Mayo Clin Proc. 2008; 83(5):554-558. 
18. Krueger K, Botermann L, Schorr SG, Griese-Mammen N, Laufs U, Schulz M. Age-related medication adherence in patients with chronic heart failure: a systematic literature review. Int J Cardiol. 2015;184:728-735.

19. Boren SA, Wakefield BJ, Gunlock TL, Wakefield DS. Heart failure self-management education: a systematic review of the evidence. Int $J$ Evid Based Healthc. 2009; 7:159-168.

20. Gwadry-Sridhar FH, Arnold JM, Zhang Y, Brown JE, Marchiori G, Guyatt G. Pilot study to determine the impact of a multidisciplinary educational intervention in patients hospitalized with heart failure. Am Heart J. 2005;150(5):982.

21. Send AF, Schwab M, Gauss A, Rudofsky G, Haefeli WE, Seidling HM. Pilot study to assess the influence of an enhanced medication plan on patient knowledge at hospital discharge. Eur J Clin Pharmacol. 2014; 70(10):1243-1250.
22. Watson PW, McKinstry B. A systematic review of interventions to improve recall of medical advice in healthcare consultations. JR Soc Med. 2009;102(6):235-243.

23. Kharod BV, Johnson PB, Nesti HA, Rhee DJ. Effect of written instructions on accuracy of self-reporting medication regimen in glaucoma patients. J Glaucoma. 2006;15(3):244-247.

24. Koelling TM, Johnson ML, Cody RJ, Aaronson KD. Discharge education improves clinical outcomes in patients with chronic heart failure. Circulation. 2005;111(2):179-185.

25. Fan $\mathrm{H}, \mathrm{Yu} \mathrm{W}, \mathrm{Zhang} \mathrm{Q}$, et al. Depression after heart failure and risk of cardiovascular and all-cause mortality: a meta-analysis. Prev Med. $2014 ; 63: 36-42$.

\section{Publish your work in this journal}

Patient Preference and Adherence is an international, peer-reviewed, open access journal that focuses on the growing importance of patient preference and adherence throughout the therapeutic continuum. Patient satisfaction, acceptability, quality of life, compliance, persistence and their role in developing new therapeutic modalities and compounds to optimize clinical outcomes for existing disease states are major areas of interest for the journal. This journal has been accepted for indexing on PubMed Central. The manuscript management system is completely online and includes a very quick and fair peer-review system, which is all easy to use. Visit http://www. dovepress.com/testimonials.php to read real quotes from published authors.

Submit your manuscript here: http://www.dovepress.com/patient-preference-and-adherence-journal 